hyper-emergent NIS caseload volumes continue to increase, comparison of current and prior caseload acuity trends may allow the individual healthcare system to better optimize for efficient resource allocation, provider coverage determination, and fair on-call provider reimbursement.

Disclosures A. Linn: None. Y. Gujrati: None. O. Qahwash: None. A. Razak: None.

\section{E-082 ENDOVASCULAR PREOPERATIVE EMBOLIZATION FOR TEMPOROMANDIBULAR JOINT REPLACEMENT SURGERY}

${ }^{1} \mathrm{~A}$ Santillan*, ${ }^{2} \mathrm{M}$ Sur, ${ }^{1} \mathrm{~J} S \mathrm{Schwarz},{ }^{1} \mathrm{~K}$ Brown, ${ }^{3} \mathrm{E}$ Rogol, ${ }^{2} \mathrm{D}$ Behrman, ${ }^{1} \mathrm{~A}$ Patsalides ${ }^{1}$ Division of Interventional Neuroradiology. Deparment of Neurosurgery, Weill Cornell Medical College, New York, NY; ${ }^{2}$ Oral and Maxillofacial Surgery, Weill Cornell Medical College, New York, NY; ${ }^{3}$ Oral and Maxillofacial Surgery, University of New Mexico Health Sciences Center, Albuquerque, NM

10.1136/neurintsurg-2019-SNIS. 157

Background and purpose This retrospective study evaluates the safety and effectiveness of preoperative endovascular embolization in patients who underwent temporomandibular joint (TMJ) replacement surgery.

Material and methods We included all patients treated with preoperative embolization of the internal maxillary artery (IMAX) between June 2016 and January 2019. All patients were treated by the same surgeon using standard surgical approaches and procedures. Periprocedural adverse events, blood loss during surgery and clinical follow-up are reported.

Results Fourteen patients (12 females, mean age 32.5) were treated with 19 embolizations of the internal maxillary artery (bilateral embolizations in 7 patients) prior to TMJ replacement surgery with prosthetic joints (TMJ Concepts prostheses). Seven patients presented with TMJ ankylosis/degenerative joint disease/post-trauma deformity, 4 patients with Idiopathic Condylar Resorption and resultant mandibular displacement/ hypoplasia, 2 patients with rheumatoid arthritis-associated condylar degeneration and resultant loss of mandibular position, and 1 patient being re-reconstructed following management of a prosthetic joint infection. Seven patients underwent bilateral prosthetic joint replacement. Four patients underwent

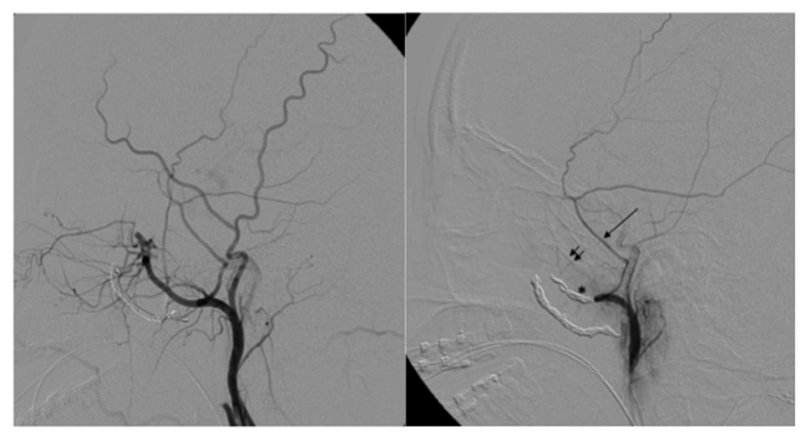

Abstract E-082 Figure 1 A 17-year-old female with past medical history of mandibular hypoplasia presenting for preoperative embolization of bilateral internal maxillary arteries. A. Pre-embolization angiogram (lateral view) of the left external carotid artery. B. The internal maxillary artery was completely embolized with coils (asterisk) with preservation of the left middle meningeal artery (MMA) (arrow) and the occluded accessory meningeal artery (AMA) got reconstituted (double arrows). Following temporomandibular joint surgery, patient had paresthesia on the left trigeminal nerve distribution more than the right at last follow-up additional facial skeletal surgery as part of their treatment. The median blood volume loss during TMJ surgery was approximately $370 \mathrm{cc}$ per patient and $246 \mathrm{cc}$ per TMJ replacement surgery (range $100 \mathrm{cc}$ to $800 \mathrm{cc}$ ). Joint space-specific blood loss was not recorded but, as per the surgical team, was significantly decreased when compared to nonembolized patients. There were no intra-procedural complications. The mean clinical follow-up was 7.1 months (range 124 months). The modified Rankin scale (mRS) was 0 before the procedure and at last clinical follow-up in all patients. After TMJ surgery, 3 patients reported paresthesia of the trigeminal nerve likely related to the residual condyle resection and 3 patients had mild facial nerve weakness (Temporal and/ or Marginal Mandibular branch) related to the surgical exposures.

Conclusion Endovascular preoperative embolization of the internal maxillary artery (IMAX) is feasible and safe and likely effective in reducing blood volume loss in complex TMJ replacement surgery.

Disclosures A. Santillan: None. M. Sur: None. J. Schwarz: None. K. Brown: None. E. Rogol: None. D. Behrman: None. A. Patsalides: None.

\section{E-083 COIL MIGRATION FOLLOWING INTERNAL CAROTID ARTERY PSEUDOANEURYSM OBLITERATION}

D Sheinberg ${ }^{*}$, D McCarthy, E Luther, S Chen, R Starke. University of Miami Miller School of Medicine, Miami, FL

\subsection{6/neurintsurg-2019-SNIS.158}

Introduction Carotid blowout syndrome (CBS) refers to the acute rupture of any segment of the common carotid artery, including both the external and extracranial internal carotid arteries (ICA). CBS often results in life threatening hemorrhage and acute upper airway obstruction. CBS is a wellknown phenomenon in patients with head and neck cancer and typically occurs via direct extension of tumor into the arterial wall or iatrogenically during surgical manipulation. With a reported incidence of $4.3 \%$ following radical neck dissection and mortality rates as high as 60\%, CBS must always be considered in any patient with head or neck cancer and acute hemorrhage in the oro- or nasopharynx of unknown origin. Accepted management strategies for CBS include open surgical ligation or endovascular treatment via parent vessel sacrifice or intraluminal stent placement. One of the more feared complications associated with endovascular parent vessel sacrifice is distal migration of embolic material, which can lead to ischemic stroke. Delayed migration of embolic material into the oro- or nasopharynx is extremely uncommon. We present a case of a coil migration into the nasopharynx one year following endovascular treatment of CBS that occurred during nasopharyngeal carcinoma resection.

Methods A 41-year-old female presented with brisk oral cavity hemorrhage after undergoing surgical resection of nasopharyngeal carcinoma seven weeks prior.

Results Computed tomography angiography (CTA) revealed a small $0.2 \times 0.2 \times 0.3 \mathrm{~cm}$ pseudoaneurysm arising from the medial aspect of the right ICA at the level of C1-C2 vertebrae that abutted the nasopharynx. After radiographically passing a balloon test occlusion (BTO), the decision was made to endovascularly sacrifice the right ICA. With the balloon inflated, embolization with coils and onyx was performed in the 